\title{
Infiltration of Natural Caries Lesions in Relation to Their Activity Status and Acid Pretreatment in vitro
}

\author{
K.W. Neuhaus ${ }^{a} \quad$ S. Schlafer ${ }^{b} \quad$ A. Lussi $^{a} \quad$ B. Nyvad ${ }^{b}$ \\ ${ }^{a}$ Department of Preventive, Restorative and Pediatric Dentistry, University of Bern, Bern, Switzerland; \\ ${ }^{b}$ Department of Dentistry, HEALTH, Aarhus University, Aarhus, Denmark
}

\section{Key Words}

Enamel caries $\cdot$ Hydrochloric acid • Lesion activity •

Nonoperative treatment $\cdot$ Phosphoric acid

\begin{abstract}
This study aimed at testing how active and inactive enamel caries lesions differ by their degree of resin infiltration, and whether the choice of acid pretreatment plays a crucial role. Four examiners assessed 104 human molars and premolars with noncavitated enamel lesions and classified them as 'active' or 'inactive' using the Nyvad criteria. Forty-five teeth were included in this study after independent unanimous lesion activity assessment. Lesions were cut perpendicularly into 2 halves. Each half lesion was pretreated with either 15\% hydrochloric acid or $35 \%$ phosphoric acid. The lesions were infiltrated after staining with rhodamine isothiocyanate. Thin sections of $100 \mu \mathrm{m}$ were prepared and the specimens were bleached with $30 \%$ hydrogen peroxide. The specimens were then counterstained with sodium fluorescein, subjected to confocal laser scanning microscopy and analyzed quantitatively. Outcome parameters were maximum and average infiltration depths as well as relative penetration depths and areas. In active lesions no significant difference of percentage maximum penetration depth and percentage average penetration depth between lesions pretreated with hydrochloric or phosphoric acid could be observed. In inactive lesions, however, phosphoric acid pretreatment resulted
\end{abstract}

in significantly lower penetration compared to hydrochloric acid pretreatment. Surface conditioning with hydrochloric acid led to similar infiltration results in active and inactive lesions. Moreover, inactive lesions showed greater variability in all assessed infiltration parameters than did active lesions. In conclusion, caries lesion activity and acid pretreatment both influenced the infiltration. The use of phosphoric acid to increase permeability of the surface layer of active lesions should be further explored.

Copyright $\odot 2012$ S. Karger AG, Basel

White spot lesions of the enamel are the first clinical signs of dental caries. Depending on the site-specific state of caries activity, the lesion might either tend to progress ('active lesion') or to stagnate or regress ('inactive lesion') [Manji et al., 1991]. Net progression of a lesion may over time lead to cavitation of the enamel surface, which makes operative intervention necessary if the cavitated area cannot be cleaned by the patient. According to clinical evidence, the risk of cavity formation is substantially higher for active lesions than for inactive lesions [Nyvad et al., 2003], which have been described to remain stable over decades [Backer Dirks, 1966]. Assessment of the activity status of caries lesions is therefore a crucial point for treatment planning [Nyvad et al., 2008].

Traditional preventive measures aim at inactivating active noncavitated enamel lesions by enhancing 'remin-

\footnotetext{
Dr. Klaus W. Neuhaus, MMA, MAS

Department of Preventive, Restorative and Pediatric Dentistry

School of Dental Medicine, University of Bern

Freiburgstrasse 7, CH-3010 Bern (Switzerland)

E-Mail klaus.neuhaus@zmk.unibe.ch
} 
eralization'. This might be achieved by better oral hygiene, including the daily use of a toothbrush and fluoride toothpaste. Professional application of high-concentrated fluoride products may boost a positive outcome [Ellwood et al., 2008; Marinho, 2008]. This strategy requires a good compliance by the patient. Patients, however, often show multiple active lesions as a consequence of a lack of compliance and neglect of oral hygiene. Thus, a more invasive chairside method has recently been suggested. This method is the so-called 'infiltration' technique, which aims at instantaneous lesion inactivation of noncavitated enamel lesions by infiltrating the porous carious enamel with a specific resin. The infiltration method was introduced in the last decade and has been tested in vitro on artificial caries of bovine teeth [Paris et al., 2006, 2007a; Meyer-Lueckel and Paris, 2008b] and on natural white spot lesions of human teeth in vitro [Paris et al., 2007b; Meyer-Lueckel and Paris, 2010]) and in vivo in adults [Paris et al., 2010; Martignon et al., 2012; MeyerLueckel et al., 2012] and children [Ekstrand et al., 2010].

Lesion inactivation by infiltration is supposed to be achieved by impregnation of the lesion body up to a depth of $620 \mu \mathrm{m}$ [Paris et al., 2009; Meyer-Lueckel and Paris, 2010] whereby dissolution of enamel by bacterial acids is hampered [Paris et al., 2010]. Importantly, it was demonstrated that in order to obtain successful infiltration of the lesion body the relatively hypermineralized surface layer of the white spot lesions has to be removed by the application of an etching procedure using 15\% hydrochloric acid for 90-120 s [Meyer-Lueckel and Paris, 2008b]. Studies reported that only after complete surface layer reduction do capillary forces allow penetration of the infiltrant into deeper parts of the lesion [MeyerLueckel and Paris, 2010]. Surface etching with phosphoric acid (20-40\%) was tested for resin infiltration in earlier studies using artificial caries models [Gray and Shellis, 2002; Paris et al., 2006] but was found inferior to pretreatment with $15 \%$ hydrochloric acid with respect to surface layer reduction [Meyer-Lueckel et al., 2007]. However, little is known about the clinical consequences of complete removal of the mineralized surface layer of incipient enamel lesions. Long-term success of the infiltration technique is likely to depend on the stability of the applied resin over time. In a randomized controlled trial, lesion progression was observed in $4 \%$ of the infiltrated proximal initial lesions after 3 years, compared to $42 \%$ in the control group [Meyer-Lueckel et al., 2012]. The control group was advised to brush the teeth twice daily with a fluoride toothpaste, to use fluoridated salt and finally to use a fluoridated gel once per week. Similarly, in a 3-year trial it was shown that infiltration or sealing of proximal surfaces had a significantly better therapeutic effect for controlling caries progression than placebo treatment in individuals who received routine instructions on flossing [Martignon et al., 2012]. While these observations suggest that infiltration of approximal caries is a promising method for caries control, there is still some concern that undetected microcavities in the enamel surface [Fejerskov et al., 2008] that cannot effectively be filled by the infiltrant [Paris et al., 2011] might hamper the long-term success of the treatment. It has therefore been proposed that to safeguard the clinical outcome of the infiltration an additional sealant at the surface could be advantageous [Wiegand et al., 2011]. A better acid resistance of infiltrated and additionally sealed artificial lesions was shown compared to infiltrated lesions alone [Schmidlin et al., 2012]. However, although perhaps desirable, it is technically demanding and time consuming to cleanly apply an additional sealant on top of an infiltrated proximal lesion.

Earlier studies of lesion infiltration were performed on lesions confined to the inner half of enamel [Paris et al., 2007b; Meyer-Lueckel and Paris, 2008a], but only recently did the inclusion criteria for white spot lesions in those studies consider their visual appearance ('dull surface, chalky opacity') [Meyer-Lueckel and Paris, 2010; Paris et al., 2011], possibly in an attempt to reflect the status of lesion activity. For purposes of caries control it may only be necessary to infiltrate active lesions, because lesions of this category have a poorer prognosis than inactive lesions [Nyvad et al., 2003]. Moreover, active lesions may be particularly receptive for infiltration because the surface layer of these lesions is more porous than that of inactive lesions [for review, see Fejerskov et al., 2008].

This study had two main goals: to test the method of infiltration in natural white spot lesions that have visually been classified as active or inactive according to the Nyvad criteria [Nyvad et al., 1999] and to test the influence of pretreatment with 35\% phosphoric acid or 15\% hydrochloric acid on infiltration of active and inactive white spot lesions.

\section{Materials and Methods}

\section{Tooth Selection}

104 human teeth (premolars, molars) were chosen from a pool of extracted teeth stored in $1 \%$ chloramine solution. Informed consent was obtained from the donors to use their teeth for scientific purposes. The chosen teeth showed initial white spot lesions on a proximal surface (ICDAS score 2) [Topping and Pitts, 2009]. 


\section{Lesion Activity Assessment}

The teeth were cleaned and photographed using a digital camera (AxioCam MRc5; Zeiss, Jena, Germany). The printouts of the photographs were used to identify the test site. Four examiners assessed the teeth independently, visually by the naked eye with/ without the aid of a stereomicroscope at $6 \times$ magnification (M531660; Wild, Heerbrugg, Switzerland), and scored them as active or inactive according to the visual-tactile criteria of Nyvad [Nyvad et al., 1999]. Lesions with a matt and rough surface were scored active, whereas lesions with a shiny and smooth surface were scored inactive. Only lesions that were unanimously scored as active and inactive by all four examiners were included in this study.

\section{Specimen Preparation}

The lesions were sectioned perpendicularly to the surface (Leica SP1600; Leica Microsystems, Wetzlar, Germany), resulting in two lesion halves. The cut surfaces were photographed at $12.5 \times$ magnification. The cut surfaces and sound enamel were covered by an acid-resistant nail varnish (Maybelline Ultra Lasting; Gemey-Maybelline, Paris, France) leaving a window around the enamel lesions. Paired lesion halves were randomly allocated (www.randomization.com) to one of two etching procedures.

\section{Surface Pretreatment, First Staining and Infiltration}

The surface of the carious enamel was pretreated with either 35\% phosphoric acid gel (Scotchbond; 3M Espe, Seefeld, Germany) or with 15\% hydrochloric acid gel (ICON-Etch; DMG, Hamburg, Germany) for $120 \mathrm{~s}$. The acidic gel was gently moved with a microbrush during application time. Subsequently, the acid gel was washed away during $30 \mathrm{~s}$ using a 3 -in-1 dental syringe. All teeth were then stored in an ethanolic solution of rhodamine B isothiocyanate (RITC 0.1\%; Sigma-Aldrich, Steinheim, Germany) for $12 \mathrm{~h}$ as described by Paris et al. [2009]. The lesions were dried by compressed air for $30 \mathrm{~s}$. A commercial infiltrant (ICON-Infiltrant, DMG) was applied on the lesion surface using a microbrush and allowed to penetrate for $3 \mathrm{~min}$. Excess infiltrant material was wiped away with dry microbrushes before light curing for $40 \mathrm{~s}$ at $1,200 \mathrm{~mW} / \mathrm{cm}^{2}$ (Bluephase; Ivoclar Vivadent, Schaan, Liechtenstein). To bleach all red fluorophores from RITC that were not enclosed by infiltrant, specimens were stored in $30 \%$ hydrogen peroxide solution for $12 \mathrm{~h}$ at $37^{\circ} \mathrm{C}$. Subsequently, specimens were washed with tap water for $60 \mathrm{~s}$.

\section{Preparation of Thin Slices and Second Staining}

After peeling off the nail varnish, the specimens were remounted in the saw (Leica SP1600; Leica Microsystems) and sections of about $200 \mu \mathrm{m}$ were obtained. The sections were polished to a final thickness of $100 \mu \mathrm{m}$ using a water-cooled rotating polishing machine (Knuth-Rotor; Struers, Copenhagen, Denmark) with silicon carbide paper up to No. 4,000 grit. To visualize porous structures such as noninfiltrated lesion parts, specimens were immersed in a $50 \%$ ethanol solution of $100 \mu \mathrm{M}$ sodium fluorescein (NaFl; Sigma-Aldrich, Brøndby, Denmark) for $3 \mathrm{~min}$. Subsequently, specimens were washed in deionized water for $10 \mathrm{~s}$.

\section{Confocal Laser Scanning Microscopy}

The thin slices were imaged with an inverted confocal laser scanning microscope (Zeiss LSM 510 META) equipped with a $10 \times$ objective, 0.3 numerical aperture (EC Plan-NEOFLUAR).
488- and 543-nm laser lines were used for sequential excitation of $\mathrm{NaFl}$ and RITC, and fluorescence emission was detected in 501544 and 597-651 nm intervals (META detector) with the confocal pin holes set to an optical slice thickness of $11.5 \mu \mathrm{m}$. For each lesion, seven equidistant $(15 \mu \mathrm{m}) \mathrm{z}$-stack images were acquired. Images were $1,024 \times 1,024$ pixels $\left(1,273 \times 1,273 \mu \mathrm{m}^{2}\right)$ in size and were acquired with pixel dwell time $1.28 \mu \mathrm{s}$, line average 2, 8-bit intensity resolution. If lesions exceeded the size of one microscopic field of view, multiple images were taken.

\section{Image Analysis}

All distance measurements were performed along the axis of the enamel rods (Image J; NIH, Bethesda, Md., USA). Average lesion depth $\left(\mathrm{LD}_{\mathrm{av}}\right)$ was calculated as the mean value of 21 randomly allocated distance measurements in all layers of the $\mathrm{z}$-stack. The maximum $\mathrm{LD}\left(\mathrm{LD}_{\max }\right)$ was defined as the greatest distance found between the surface and bottom of the lesion in any of the 7 stack layers. Accordingly, penetration depth (PD) of the infiltrant was measured as maximum $\left(\mathrm{PD}_{\max }\right)$ and average $\mathrm{PD}\left(\mathrm{PD}_{\mathrm{av}}\right)$, using the same measurement points as for LD. Furthermore, maximum projections of the acquired $\mathrm{z}$-stacks were produced (Image J), and for every lesion the relative infiltrated area was calculated using the daime software (digital image analysis in microbial ecology) [Daims et al., 2006].

\section{Statistical Analysis}

Statistical analysis was performed using SPSS software (PASW for Mac 17.0; SPSS Inc., Chicago, Ill., USA). Differences in LD between active and inactive lesions were calculated using the Wilcoxon signed rank test. To more accurately estimate the infiltration effectiveness, $\mathrm{PD}_{\max }$ and $\mathrm{PD}_{\mathrm{av}}$ were calculated relative to $\mathrm{LD}$ $\left(\% \mathrm{PD}_{\max }, \% \mathrm{PD}_{\mathrm{av}}\right)$. To test the impact of the activity status or acid pretreatment on relative infiltration area, $\% \mathrm{PD}_{\max }$ and $\% \mathrm{PD}_{\mathrm{av}}$, a nonparametric ANOVA model with repeated measurements, i.e. the F1_LD_F1 model [Brunner et al., 2002], was applied. Post hoc descriptive analyses were applied to compare the acids per activity status and the activity status per acid (Wilcoxon rank sum test). The level of significance was set at 0.05 for all tests.

\section{Results}

In the sample of 104 teeth with noncavitated enamel caries, many lesions were 'mixed lesions', containing both active and inactive sites. Twenty-one lesions were unanimously scored as 'typical active' and 24 lesions as 'typical inactive'; thus, the final sample consisted of 45 lesions. No significant differences regarding $\mathrm{LD}_{\max }$ could be observed for the corresponding lesion halves that were treated with either phosphoric or hydrochloric acid (Wilcoxon signed rank test; $\mathrm{p}>0.05$ ).

Median values and the corresponding nonparametric 95\% CI of LD and PD are given in table 1. Inactive lesions were shallower than active lesions (table 1). However, in inactive lesions the phosphoric acid etching procedure seemed to result in enough open enamel pores to allow 

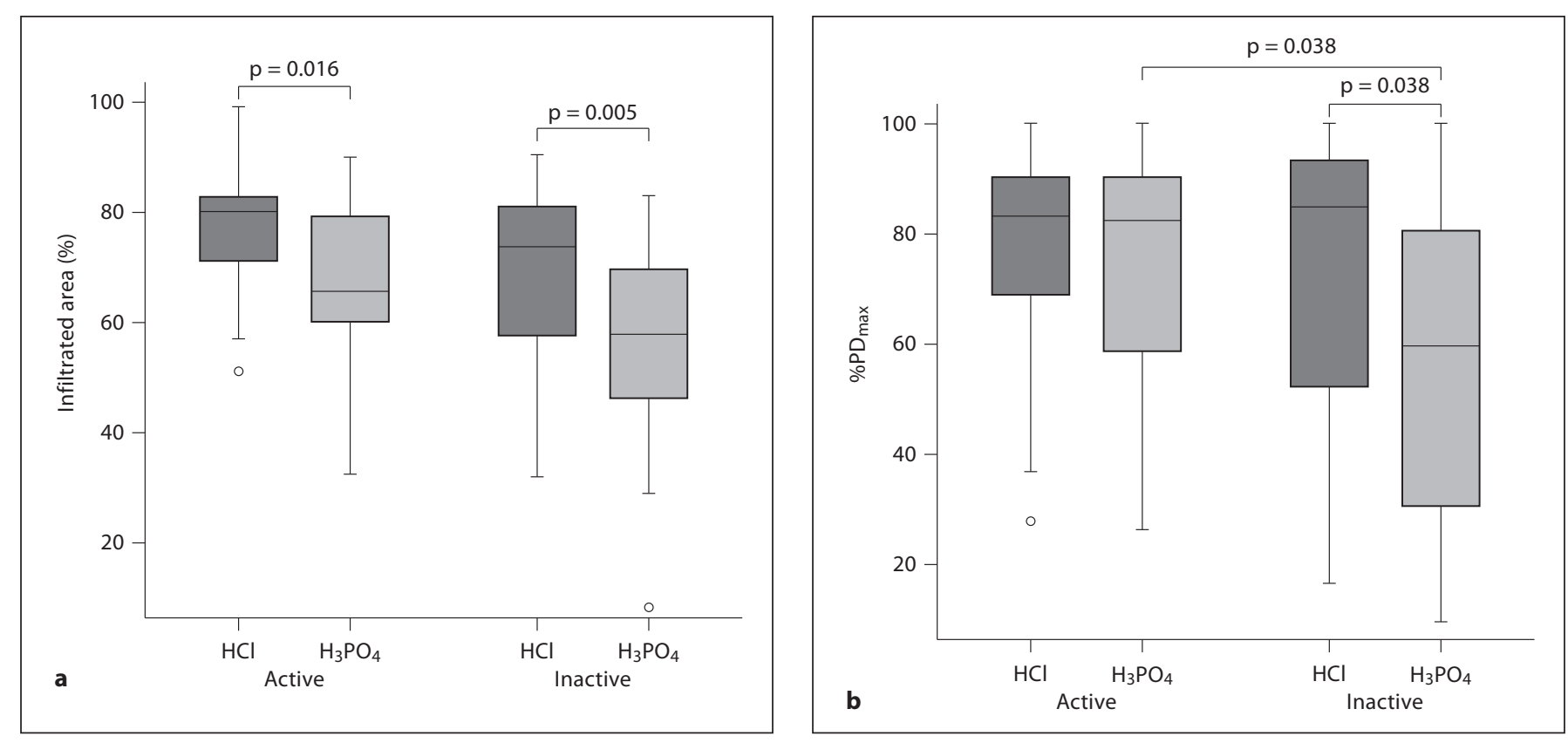

Fig. 1. Infiltration of active and inactive lesions pretreated with $35 \%$ phosphoric acid $\left(\mathrm{H}_{3} \mathrm{PO}_{4}\right)$ or $15 \%$ hydrochloric acid $(\mathrm{HCl})$. Significant differences are indicated. The comparisons between active lesions pretreated with $\mathrm{HCl}$ and inactive lesions pretreated with $\mathrm{H}_{3} \mathrm{PO}_{4}$ were highly significant $(\mathrm{p}<0.01)$. a Relative infiltrated areas in maximum projection images of the lesions. b $\% \mathrm{PD}_{\max }$ of the infiltrant. c $\% \mathrm{PD}_{\mathrm{av}}$ of the infiltrant.

infiltration into the lesion body up to about $300 \mu \mathrm{m}$ or about $60 \%$ of the lesion area (table 1; fig. 1a).

Both lesion activity status and the chosen type of acid pretreatment had a significant effect on the relative infiltrated area, while $\% \mathrm{PD}_{\max }$ was significantly influenced by acid pretreatment and $\% \mathrm{PD}_{\mathrm{av}}$ by lesion activity only (table 2). The product factor 'activity:acid' describes the interaction of acid and lesion activity, i.e. whether the ef-

fect of the acid pretreatment is limited to active or inactive lesions. No such effect could be detected (table 2).

For visualization, the quantitative results for $\% \mathrm{PD}_{\max }$, $\% \mathrm{PD}_{\mathrm{av}}$, and relative infiltrated areas are presented as boxplots in figure $1 . \% \mathrm{PD}_{\max }$ did not differ between active lesions pretreated with hydrochloric or phosphoric acid and inactive lesions pretreated with hydrochloric acid, but $\% \mathrm{PD}_{\max }$ was significantly lower in inactive lesions 

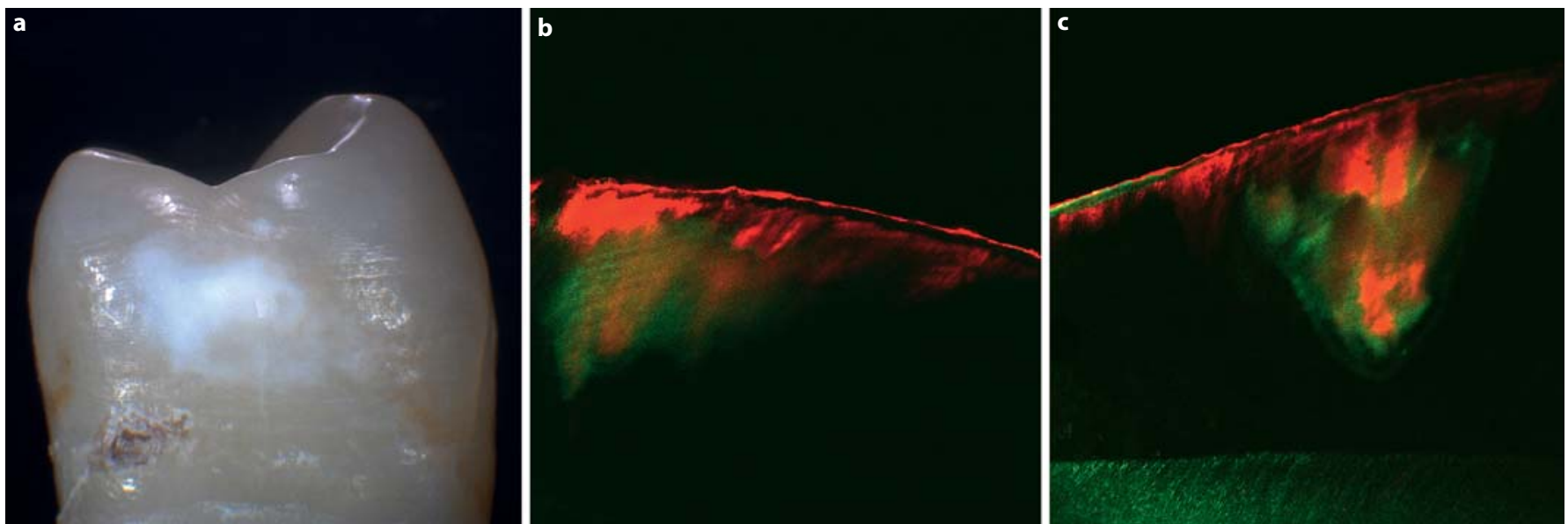

Fig. 2. Lesion unanimously rated as 'active'. a Photograph, $12.5 \times$ magnification. b Lesion half, infiltrated after pretreatment with $15 \%$ hydrochloric acid. The infiltrated part of the lesion is stained with RITC (red), while noninfiltrated areas are stained with so- dium fluorescein (green). c Lesion half, infiltrated after pretreatment with $35 \%$ phosphoric acid. $\% \mathrm{PD}_{\max }$ and $\% \mathrm{PD}_{\mathrm{av}}$ did not differ between the two acid pretreatments.

Table 1. Median values (and corresponding nonparametric $95 \% \mathrm{CIs}$ ) of $\mathrm{LD}_{\max }, \mathrm{LD}_{\mathrm{av}}, \mathrm{PD}_{\max }$ and $\mathrm{PD}_{\mathrm{av}}$ of active and inactive white spot lesions

\begin{tabular}{llllll}
\hline & \multicolumn{3}{l}{ Active lesions } & & \multicolumn{2}{l}{ Inactive lesions } \\
\cline { 2 - 3 } \cline { 5 - 6 } & $\mathrm{HCl}$ & $\mathrm{H}_{3} \mathrm{PO}_{4}$ & & $\mathrm{HCl}$ & $\mathrm{H}_{3} \mathrm{PO}_{4}$ \\
\hline $\mathrm{LD}_{\max }, \mu \mathrm{m}$ & $756(630 ; 970)$ & $752(528 ; 918)$ & & $593(398 ; 879)$ & $687(469 ; 874)$ \\
$\mathrm{LD}_{\mathrm{av}}, \mu \mathrm{m}$ & $579(464 ; 742)$ & $534(402 ; 731)$ & & $479(383 ; 735)$ & $493(414 ; 626)$ \\
$\mathrm{PD}_{\max }, \mu \mathrm{m}$ & $549(376 ; 815)$ & $475(303 ; 763)$ & & $340(290 ; 539)$ & $296(251 ; 342)$ \\
$\mathrm{PD}_{\mathrm{av}}, \mu \mathrm{m}$ & $460(288 ; 742)$ & $462(261 ; 731)$ & & $288(256 ; 416)$ & $267(217 ; 340)$ \\
\hline
\end{tabular}

$\mathrm{HCl}=$ Hydrochloric acid; $\mathrm{H}_{3} \mathrm{PO}_{4}=$ phosphoric acid.

pretreated with phosphoric acid. Generally, inactive lesions showed a higher variability than active lesions with respect to all lesion parameters evaluated (fig. 1).

Figures 2 and 3 show representative examples of an active and inactive lesion that were cut in half and pretreated with different acids. Examination revealed that noninfiltrated parts of the lesions were regularly found in the innermost regions of the lesions, adjacent to sound enamel. Furthermore, many lesions showed double stained areas in single planes of a $\mathrm{z}$-stack (fig. 4).

\section{Discussion}

This study shows that both lesion activity status and acid pretreatment influence the outcome of infiltration studies of noncavitated caries lesions.

Infiltration of Active/Inactive Lesions
Table 2. Influence of activity status, acid pretreatment and the interaction between acid and activity on infiltration.

\begin{tabular}{lll}
\hline Effect & Outcome parameter & $\mathrm{p}$ value \\
\hline Activity & relative infiltrated area & 0.0109 \\
& $\% \mathrm{PD}_{\max }$ & n.s. \\
& $\mathrm{PD}_{\mathrm{av}}$ & 0.0016 \\
Acid & relative infiltrated area & $<0.0001$ \\
& $\mathrm{PD}_{\max }$ & 0.0120 \\
& $\mathrm{PD}_{\mathrm{av}}$ & n.s. \\
Activity:acid & relative infiltrated area & n.s. \\
& $\mathrm{PD}_{\max }$ & n.s. \\
& $\mathrm{PD}_{\mathrm{av}}$ & n.s. \\
\hline
\end{tabular}

ANOVA-type statistic [Brunner et al., 2002]; degree of free$\operatorname{dom}=1$.

Caries Res 2013;47:203-210 

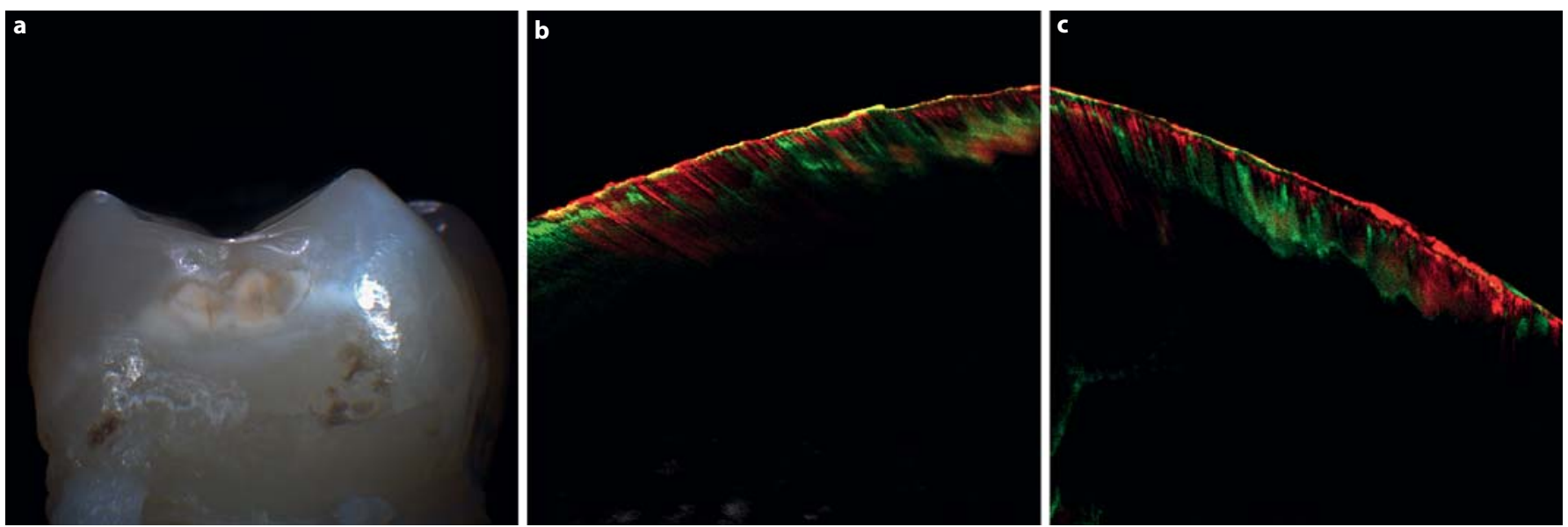

Fig. 3. Lesion unanimously rated as 'inactive'. a Photograph, $12.5 \times$ magnification. $\mathbf{b}$ Lesion half, infiltrated after pretreatment with $15 \%$ hydrochloric acid. The infiltrated part of the lesion is stained with RITC (red), while noninfiltrated areas are stained

with sodium fluorescein (green). c Lesion half, infiltrated after pretreatment with $35 \%$ phosphoric acid. Relative infiltration is smaller than after pretreatment with hydrochloric acid.

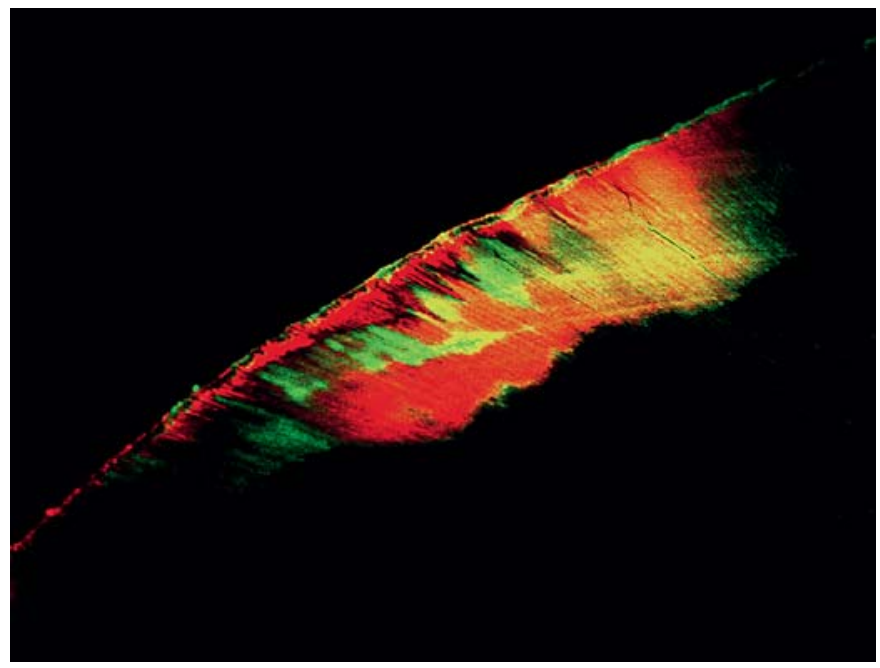

Fig. 4. Single plane of a $\mathrm{z}$-stack (confocal laser scanning microscopy) of an infiltrated lesion. We often noticed yellow areas which indicate double staining with both dyes. gesting that surface conditioning with phosphoric acid is sufficient to increase permeability of the surface layer and allow infiltration of active noncavitated lesions. By contrast, surface conditioning with phosphoric acid was less effective than hydrochloric acid in infiltration of inactive lesions. This might probably be explained by the variable and often thick surface layers of inactive lesions inhibiting penetration of acids and acrylate molecules into the lesion [Iijima and Tagaki, 2000; Cochrane et al., 2012], a phenomenon that was also reflected by a high variation in infiltration depths of the inactive lesions in our study. In a clinical perspective, our results may explain why it is more difficult to predict the outcome of postorthodontic mask- ing of inactive enamel lesions [Neuhaus et al., 2010] compared to active lesions [Paris and Meyer-Lueckel, 2009; Rocha Gomes Torres et al., 2011].

Our study applied area estimates as a response variable. These area estimates are based on maximum projections of $\mathrm{z}$-stacks of about $100-\mu \mathrm{m}$ thickness of the lesion and differ from those used in an earlier publication [Paris et al., 2011]. Previous studies of lesion infiltration have mostly been evaluated by measuring PD at the deepest site, or 
at 'characteristic' landmarks of the lesion in a single reference plane [Paris et al., 2009, 2011]. However, it has not been reported so far whether the depth measurements were taken along the enamel rods or perpendicular to the surface. The effect of acid pretreatment was shown to have a significant influence on $\% \mathrm{PD}_{\max }$ but not $\% \mathrm{PD}_{\mathrm{av}}$, while lesion activity significantly influenced $\% \mathrm{PD}_{\mathrm{av}}$ but not $\% \mathrm{PD}_{\max }$ (table 2). Area measurements could be more robust than depth measurements because a higher amount of data is included in the estimates. However, because shallow lesions are better infiltrated than deeper ones, area estimates may suggest almost complete penetration while deep lesion parts are only partially infiltrated. We found that area estimates seemed to be less dependent on penetration peaks. Therefore, giving results for both $\% \mathrm{PD}_{\max }$ and relative infiltrated areas is probably the most accurate way to report findings of in vitro infiltration studies.

PDs of the infiltrant of about $500 \mu \mathrm{m}$ in active lesions are in the range of data reported by other groups. Both lower $(264 \mu \mathrm{m})$ [Meyer-Lueckel and Paris, 2008a] and higher PDs (626 $\mu \mathrm{m}$ for the experimental resin PC204) [Meyer-Lueckel and Paris, 2010] were reported previously. A lower infiltration depth $(<50 \mu \mathrm{m})$ was found after 120 s surface pretreatment with $37 \%$ phosphoric acid [Paris et al., 2007b]. We found consistently higher infiltration depths also in inactive lesions. This might be attributable to the fact that in the latter study a more viscous resin was used which was labeled with $0.1 \%$ tetramethylrhodamine isothiocyanate. Both the resin with the lower penetration coefficient and the direct staining method might contribute to lower PD measurements. The indirect staining method that was used in the current study setup (1st stain, infiltration, bleaching, 2nd stain) showed a generally better reliability [Paris et al., 2009] and allowed more accurate resin penetration measurements that led to higher values [Paris et al., 2011].

Contrary to other studies that reported almost complete LD infiltration [Meyer-Lueckel and Paris, 2010], we only observed incomplete infiltration of about $80 \%$ of the area in active lesions and $75 \%$ in inactive lesions (fig. 1a). The deepest parts of our noncavitated lesions were typically not infiltrated (fig. 2b, c). Based on comparisons with polarized light microscopic images of incipient enamel lesions these inner parts of the lesions may correspond to the dark zone [Teranaka et al., 1986]. The dark zone has a higher content of organic material (protein and lipids) that may occlude submicroscopic pores [Shellis et al., 2002] and hamper infiltration. A recent study combined polarized light microscopy with fluorescence microscopy, and although assessment of the dark zone was not the primary aim, one superimposed figure in that publication supports our hypothesis [Arnold and Gaengler, 2012]. However, considering that in the latter study only 5 teeth were included, a firm association between the noninfiltrated area and the presence of a dark zone can only be verified in a combined experimental setup using polarized light microscopy and confocal laser scanning microscopy.

In our study, double stained areas (red and green) could be observed in both active and inactive lesions (fig. 4). One possible explanation for this phenomenon could be prior demineralization/remineralization cycles that led to irregular zones of mineral reprecipitation [ten Cate et al., 2008], hindering complete penetration of the infiltrant and leaving binding sites for $\mathrm{NaFl}$ in close proximity to red fluorophores. However, red and green fluorophores might also bind in identical xy positions, but different $\mathrm{z}$ positions of an image stack.

In conclusion, within the limitations of this laboratory study, we showed that both lesion activity status and acid pretreatment influence the depth and area of infiltration of natural white spot lesions. Consequently, lesion activity should be considered in future infiltration studies. Acid pretreatment with hydrochloric acid improved the overall lesion infiltration compared to pretreatment with phosphoric acid, especially in inactive lesions. The clinical implications of the latter finding remain speculative and leave room for discussion. From a biological point of view, it may be risky to lose the outer protective layer of an incipient lesion due to acid conditioning because if the infiltrated resin is degraded over time (Van Landuyt et al., 2011) the lesion might be prone to progression. On the other hand, we do not know if full penetration of the infiltrant into a caries lesion is of vital importance for longlasting clinical results. It could well be (as with occlusal sealing) that a firm surface seal, preventing bacterial acids from reaching the enamel, is sufficient to control lesion progression [Martignon et al., 2012]. Therefore, to protect the natural surface layer of lesions, we recommend that the use of phosphoric acid to increase permeability of the surface layer of active lesions should be further explored.

\section{Acknowledgments}

We would like to thank Mrs. Lene Grønkjær for her priceless effort in the preparation of the specimens. We would also like to thank Dr. Irene Dige for participating in the lesion activity assessment prestudy. The help of Dr. Merete K. Raarup in assistance and technical support with the confocal laser scanning microscopy is also acknowledged. Statistical analysis was performed by Jasmin 
Wandel, Institute for Mathematical Statistics and Actuarial Science of the University of Bern, which is hereby greatly acknowledged. This study was supported by a grant of the Swiss National Foundation (IZK0Z3_135913), which allowed a 3-month research fellowship at Aarhus University. We also thank DMG for support of this study with material. The funders had no role in study design, data collection and analysis, decision to publish, or preparation of the manuscript.
K.W.N., A.L. and B.N. conceived and designed the experiments; K.W.N. and S.S. performed the experiments; K.W.N., S.S., A.L. and B.N. analyzed the data; K.W.N., S.S., A.L. and B.N. wrote the manuscript.

\section{Disclosure Statement}

None of the authors of the present paper has a conflict of interest.

\section{References}

-Arnold WH, Gaengler P: Light- and electronmicroscopic study of infiltration of resin into initial caries lesions - a new methodological approach. J Microsc 2012;245:26-33.

Backer Dirks O: Posteruptive changes in dental enamel. J Dent Res 1966;45:503-511.

Brunner E, Domhof S, Langer F: Nonparametric Analysis of Longitudinal Data in Factorial Experiments. New York, Wiley, 2002.

Cochrane NJ, Anderson P, Davis GR, Adams GG, Stacey MA, Reynolds EC: An X-ray microtomographic study of natural white-spot lesions. J Dent Res 2012;91:185-191.

Daims H, Lucker S, Wagner M: Daime, a novel image analysis program for microbial ecology and biofilm research. Environ Microbiol 2006;8:200-213.

Ekstrand KR, Bakhshandeh A, Martignon S: Treatment of proximal superficial caries lesions on primary molar teeth with resin infiltration and fluoride varnish versus fluoride varnish only: efficacy after 1 year. Caries Res 2010;44:41-46.

Ellwood R, Fejerskov O, Cury J, Clarkson B: Fluorides in caries control; in Fejerskov O, Kidd EA (eds): Dental Caries - The Disease and Its Clinical Management. Oxford, Blackwell Munksgaard, 2008, pp 287-328.

Fejerskov O, Nyvad B, Kidd EA: Pathology of dental caries; in Fejerskov O, Kidd EA (eds): Dental Caries - The Disease and Its Clinical Management. Oxford, Blackwell Munksgaard, 2008, pp 19-48.

Gray GB, Shellis P: Infiltration of resin into white spot caries-like lesions of enamel: an in vitro study. Eur J Prosthodont Restor Dent 2002;10:27-32.

- Iijima Y, Takagi O: In situ acid resistance of in vivo formed white spot lesions. Caries Res 2000;34:388-394.

-Manji F, Fejerskov O, Nagelkerke NJ, Baelum V: A random effects model for some epidemiological features of dental caries. Community Dent Oral Epidemiol 1991;19:324-328.

-Marinho CV: Evidence-based effectiveness of topical fluorides. Adv Dent Res 2008;20:3-7.

Martignon S, Ekstrand KR, Gomez J, Lara JS, Cortes A: Infiltrating/sealing proximal caries lesions: a 3-year randomized clinical trial. J Dent Res 2012;91:288-292.

-Meyer-Lueckel H, Bitter K, Paris S: Randomized controlled clinical trial on proximal caries infiltration: three-year follow-up. Caries Res 2012;46:544-548.
Meyer-Lueckel H, Paris S: Improved resin infiltration of natural caries lesions. J Dent Res 2008a;87:1112-1116.

Meyer-Lueckel H, Paris S: Progression of artificial enamel caries lesions after infiltration with experimental light curing resins. Caries Res 2008b;42:117-124.

Meyer-Lueckel H, Paris S: Infiltration of natural caries lesions with experimental resins differing in penetration coefficients and ethanol addition. Caries Res 2010;44:408-414.

-Meyer-Lueckel H, Paris S, Kielbassa AM: Surface layer erosion of natural caries lesions with phosphoric and hydrochloric acid gels in preparation for resin infiltration. Caries Res 2007;41:223-230.

Deuhaus KW, Graf M, Lussi A, Katsaros C: Late infiltration of post-orthodontic white spot lesions. J Orofac Orthop 2010;71:442-447.

Nyvad B, Fejerskov O, Baelum V: Visual-tactile caries diagnosis; in Fejerskov O, Kidd EA (eds): Dental Caries - The Disease and Its Clinical Management. Oxford, Blackwell Munksgaard, 2008, pp 49-68.

Nyvad B, Machiulskiene V, Baelum V: Reliability of a new caries diagnostic system differentiating between active and inactive caries lesions. Caries Res 1999;33:252-260.

Nyvad B, Machiulskiene V, Baelum V: Construct and predictive validity of clinical caries diagnostic criteria assessing lesion activity. J Dent Res 2003;82:117-122.

-Paris S, Bitter K, Naumann M, Dorfer CE, Meyer-Lueckel H: Resin infiltration of proximal caries lesions differing in ICDAS codes. Eur J Oral Sci 2011;119:182-186.

Paris S, Bitter K, Renz H, Hopfenmuller W, Meyer-Lueckel H: Validation of two dual fluorescence techniques for confocal microscopic visualization of resin penetration into enamel caries lesions. Microsc Res Tech 2009;72: 489-494.

Paris S, Hopfenmuller W, Meyer-Lueckel H: Resin infiltration of caries lesions: an efficacy randomized trial. J Dent Res 2010;89:823-826.

Paris S, Meyer-Lueckel H: Masking of labial enamel white spot lesions by resin infiltration - a clinical report. Quintessence Int 2009;40:713-718.

- Paris S, Meyer-Lueckel H, Colfen H, Kielbassa AM: Penetration coefficients of commercially available and experimental composites intended to infiltrate enamel carious lesions. Dent Mater 2007a;23:742-748.
Paris S, Meyer-Lueckel H, Kielbassa AM: Resin infiltration of natural caries lesions. J Dent Res 2007b;86:662-666.

Paris S, Meyer-Lueckel H, Mueller J, Hummel M, Kielbassa AM: Progression of sealed initial bovine enamel lesions under demineralizing conditions in vitro. Caries Res 2006;40: 124-129.

Rocha Gomes Torres C, Borges AB, Torres LM, Gomes IS, de Oliveira RS: Effect of caries infiltration technique and fluoride therapy on the colour masking of white spot lesions. J Dent 2011;39:202-207.

-Schmidlin PR, Sener B, Attin T, Wiegand A: Protection of sound enamel and artificial enamel lesions against demineralisation: caries infiltrant versus adhesive. J Dent 2012;40:851856.

Shellis RP, Hallsworth AS, Kirkham J, Robinson C: Organic material and the optical properties of the dark zone in caries lesions of enamel. Eur J Oral Sci 2002;110:392-395.

ten Cate JM, Larsen MJ, Pearce EI, Fejerskov O: Chemical interactions between the tooth and oral fluids; in Fejerskov O, Kidd EA (eds): Dental Caries - The Disease and Its Clinical Management. Oxford, Blackwell Munksgaard, 2008, pp 209-232.

Teranaka T, Koulourides T, Butler WT: Protein content and amino-acid content of consolidated carious lesions in human enamel and of experimental lesions in bovine enamel exposed to the human mouth. Arch Oral Biol 1986;31:405-410.

Thylstrup A, Bruun C, Holmen L: In vivo caries models - mechanisms for caries initiation and arrestment. Adv Dent Res 1994;8:144157.

Topping GV, Pitts NB: Clinical visual caries detection. Monogr Oral Sci 2009;21:15-41.

-Van Landuyt KL, Nawrot T, Geebelen B, De Munck J, Snauwaert J, Yoshihara K, Scheers H, Godderis L, Hoet P, Van Meerbeek B: How much do resin-based dental materials release? A meta-analytical approach. Dent Mater 2011;27:723-747.

Wiegand A, Stawarczyk B, Kolakovic M, Hämmerle CH, Attin T, Schmidlin PR: Adhesive performance of a caries infiltrant on sound and demineralised enamel. J Dent 2011;39: 117-121. 